\title{
J. Therapy of Intracranial Aneurysm
}

\author{
J-1. Artificial Embolization of Steel Ball for \\ Intracranial Arterio-Venous Malformations with the \\ Aid of a Giant Extracranial Electric Magnet
}

\author{
Ryuichi Tsugane, M. D., Kenichiro Sugita, M. D., \\ Naomi Mutsuga, M. D., and Akinari Dor, M. D. \\ Department of Neurosurgery, Nagoya University School of Medicine
}

Artificial embolization has been applied in the management of large and inoperable intracranial arteriovenous malformations by several investigators. The accurate placement of the emboli still remains the problem. In the usual embolizing method, embolus which was introduced into the carotid artery flows into the middle cerebral artery and the malformations located mainly in the anterior cerebral artery territory have not been embolized.

In an attempt to embolize the malformation in the anterior cerebral artery area, a huge electric magnet for ophthalmology was applied for pulling in the embolus into the anterior cerebral artery. The model experiments were performed on " $Y$ " and " $T$ " shaped glass tube under the various flow rates of water. The steel balls of 1 to $3 \mathrm{~mm}$ in diameter could be safely pulled into sideways at 900 Gauss or more. The maximum capacity of the magnent (100V-30 Amp) was 1500 Gauss at the distance of $5 \mathrm{~cm}$ from the head of the magnent and 600 Gauss at $10 \mathrm{~cm}$. The animal experiments were performed on dogs embolizing the renal artery under the control of the magnet. The results were satisfactory.

Clinical experiences are consisted of four arteriovenous malformations fed from both the anterior and middle cerebral arteries and one malformation located in basal ganglia fed from the dilated lenticulosteriate arteries. In the anterior and middle cerebral artery group, the magnet was placed at the fronto-temporal area, not to exceed $8 \mathrm{~cm}$ from the bifurcation of the internal carotid artery. In a case of the basal ganglia, the magnet was placed at the vertex directing to the base of the skull. The size of the steel ball was decided according to the diameter of feeding artery. In the first three cases, non-coated steel balls were used. In latter cases, silicon coated steel balls were used to avoid inadequate vascular responses to the emboli.

84 to $95 \%$ of the steel balls embolized the aimed arteries and the other 16 to $5 \%$ flew into the feeding arteries from the middle cerebral artery. In each cases one to three balls embolized the arteries to the normal brain. No case showed the permanent sign due to the mislocation of the emboli. We have no experience of a case in which the emboli passed the malformation and flew into the venous circulation. In two cases the diminishment of the malformation on serial angiogram was almost complete and in the other three cases it was moderately accomplished. But the follow-up angiography revealed newly formed collateral circulation and visualization of the 
malformation again. In three cases radical removal of the malformation was performed about 1 to 3 months after embolization. In each case, removal of the malformation was successfully performed without massive hemorrhage and the feeding arteries were easily found because of the location of the embolized.

The authers present concept to the artificial embolization is that the embolization may have some part in the preoperative management of the arterio-venous malformation and in future, if three pairs of the more powerful magnets are available for clinical use with controling system of the magnet and tracing mechanism of the embolus, many other inoperable malformation will be embolized by steel balls.

\title{
J-2. Stereotaxic Thrombosis of Intracranial Aneurysm
}

\author{
Nobuo Yoshir, Kashiwa Mrkami and Kinya Oshida \\ Division of Neurological Surgery, Department of Surgery, \\ School of Medicine, Keio University
}

The major factors in the motality and mobidity of early surgery for aneurysm appear to be the vasospasmums and subsequent infarction that follow surgical manipulation of the already irritated cerebral blood vessels, and the edema and contusion of the brain that result from operative retraction. Stereotaxic surgery is ideally suited to overcome these disadvantages. A stereotaxic probe can be placed at any point within the head without brain retraction. With X-ray control, a probe can be positioned directly against the dome of an aneurysm without dissection or manipulation of arteries of the circle of Willis. We have modified Alksne's technique by placing a small magnet directly against the aneurysm. A 7 gauge needle is tip protrudes $4 \mathrm{~mm}$ beyond the end of the probe and penetrate the wall of the aneurysm. Iron suspension is injected into the aneurysmal fund. The magnetic field holds the iron in place as it serves as a nidus for blood coagulation. Experimental study is performed and thrombosis of artificial aneurysm by injecting carbonyl iron suspension with glycerin or gelatin solution (instead of $20 \%$ dextran, Alksne). One of the problem is settling of iron powder in the syringe every two minutes during facilitate slow continuous injection. $10 \%$ gelatin solution prevents settling of iron powder about one hour or so. Iron suspension with glycerin prevent setting or iron powder for long time. Effects of ultrasonic vibration is remarkable for mixture of the suspension in the syringe. From these facts we have used carbonyl iron covered with gelatin molecule in the human albumin as a suspension. Ultrasonic vibration is also used mixture of the suspension intermittently. Animal experiment and one case of human are succesfully done with our technique. 